\title{
THE NULLITY SPACES OF CURVATURE-LIKE TENSORS
}

\author{
ROBERT MALTZ
}

\section{Introduction}

In this paper we use the methods of the author [7] to prove the following general theorem on the nullity spaces of tensor fields which have all the formal properties of the curvature tensor. This result may be regarded as an intrinsic version of the immersion theorem proved in [7]. See $\S 2$ for precise definitions.

Theorem 1. Let $K$ denote a curvature-like tensor on a Riemannian manifold $M$, and $\mu_{K}$ its index of nullity. Assume $M$ is complete, and let $G_{K}$ be the open set on which $\mu_{K}$ takes its minimum value $m$ (assumed $>0$ ). Then every leaf $L$ of the nullity foliation of $K$ induced on $G_{K}$ is complete (and totally geodesic in $M$ ).

This theorem generalizes results given in Maltz [6] and Clifton-Maltz [3], and our proof, besides being considerably simpler, corrects an expository error in those papers. Our result also includes recent results of Abe [1] (given here as Corollaries 1, 2 and parts of Corollaries 3 and 4), obtained by refining the proofs in [6] and [3].

In $\S 3$ we consider some well-known examples of curvature-like tensors, obtaining the following results as corollaries of Theorem 1 .

Corollary 1. Let $M$ be a complete Riemannian manifold, and let $K_{X Y}=$ $R_{X Y}-k(X \wedge Y)$. Then the leaves of the nullity foliation of $K$ induced on $G_{K}$ are complete totally geodesic submanifolds with constant curvature $k$.

Corollary 2. Let $M$ be a complete Kählerian manifold, and let $K_{X Y}=$ $R_{X Y}-(k / 4)(X \wedge Y+J X \wedge J Y+2\langle X, J Y\rangle J)$. Then the leaves of the nullity foliation of $K$ induced on $G_{K}$ are totally geodesic submanifolds with constant holomorphic curvature $k$.

The following result gives a second class of examples.

Theorem 2. Let $I: M \rightarrow \bar{M}$ be an isometric immersion of $M$ into another Riemannian manifold $\bar{M}$. Suppose that $P^{\perp} \bar{R}_{X Y} Z=0$ for all vector fields $X, Y$, $Z$ tangent to $M$ ( $X$ is identified with $I_{*} X$, etc., as in [7]; $P^{\perp}$ denotes projection normal to $M$ ). Then the curvature difference tensor $D_{X Y}=R_{X Y}-\bar{R}_{X Y}$ on $M$ is curvature-like.

It is interesting to note that the condition $P \perp \bar{R}_{X Y} Z=0$ is precisely what was needed to prove the immersion theorem of [7], so this type of immersion has particularly nice properties. Examples are provided by immersions into

Communicated by H. Samelson, April 12, 1971. 
spaces $\bar{M}$ of constant curvature or by Kähler immersions of a Kähler manifold $M$ into a Kähler manifold $\bar{M}$ of constant holomorphic curvature.

\section{The nullity spaces of a curvature-like tensor}

Let $M$ be a $C^{\infty}$ Riemannian manifold. A $(1,3)$ tensor field $K$ on $M$ is said to be curvature-like, if it has all the formal properties of the curvature operator. Hence, using the notational conventions of [7], given any $C^{\infty}$ manifold $L$ and $C^{\infty}$ map $F: L \rightarrow M, K$ satisfies

i) $\left\langle K^{*}{ }_{A_{*} B_{*}}(X), Y\right\rangle=-\left\langle K^{*}{ }_{A_{*} B_{*}}(Y), X\right\rangle$,

ii) $K^{*}{ }_{A_{*} B_{*}}=-K_{B_{*} A_{*}}$,

iii) $\left\langle K^{*}{ }_{A_{*} B_{*}}\left(C_{*}\right), D_{*}\right\rangle=\left\langle K^{*}{ }_{C_{*} D_{*}}\left(A_{*}\right), B_{*}\right\rangle$,

iv) $\subseteq K^{*}{ }_{A_{*} B_{*}}\left(C_{*}\right)=0$,

v) $\mathbb{S}\left(\nabla_{A} K^{*}\right)_{B_{*} C_{*}}=0$,

where $A, B, C, D \in \mathfrak{X}(L) ; X, Y \in \mathfrak{X}_{F}(M)$; and $\subseteq$ denotes cyclic summation over $A, B$, and $C$. iii) actually follows from i), ii), and iv), but otherwise these conditions are independent.

The following alternative version of v) will be needed later.

Lemma 1. $\widetilde{S} \nabla_{A}\left(K^{*}{ }_{B_{*} C_{*}}\right)=\subseteq S^{*}{ }_{[A, B]_{*}, C_{*}}$. In particular, if $[A, B]=[A, C]=$ $[B, C]=0$, then $\subseteq \nabla_{A}\left(K_{B_{*} C_{*}}\right)=0$.

Proof. We have $[A, B]_{*}=\nabla_{A} B_{*}-\nabla_{B} A_{*}$, etc., by the generalized second structural equation, [7]. Using these relations and v), we have $\mathfrak{S} \nabla_{A}\left(K_{B_{*} C_{*}}\right)=$ $\mathfrak{S}\left(\left(\nabla_{A} K\right)_{B_{*} C_{*}}+K_{\nabla_{A} B_{*}, C_{*}}+K_{B_{*}, \nabla_{A} C_{*}}\right)=\mathfrak{S}\left(K_{\nabla_{A} B_{*}, C_{*}}+K_{C_{*}, \nabla_{B} A_{*}}\right)=\mathfrak{S}\left(K_{[A, B]_{*}, C_{*}}\right)$.

For each $p \in M$, set $N_{K}(p)=\left\{x \in M_{p}: K_{x y}=0\right.$ for all $\left.y \in M_{p}\right\} . N_{K}(p)$ is the nullity space of $K$ at $p . N_{K}(p)$ is a linear subspace of $M_{p}$, and its dimension will be denoted by $\mu_{K}(p)$, the index of nullity of $K$ at $p . \mu_{K}$ is upper-semicontinuous, so the set $G_{K}$ on which it assumes its minimal value $m$ is open.

By writing out all the terms in $\subseteq \nabla_{A}\left(K_{B C} D\right)=\subseteq\left(\left(\nabla_{A} K\right)_{B C} D+K_{B C}\left(V_{A} D\right)+\right.$ $\left.K_{\nabla_{A} B, C} D+K_{B, \nabla_{A} C} D\right)$, where $A, D$ are nullity vector fields $\left(A_{p} \in N_{K}(p)\right.$, etc.), and $B, C$ are arbitrary vector fields on $M$, we find that $K_{B C}\left(\nabla_{A} D\right)=0$. By the antisymmetries of $K$, this implies that $\nabla_{A} D$ is a nullity vector field (we write $\nabla_{A} D \in N_{K}$ ) whenever $A$ and $D$ are. It follows as in [7] that the nullity of $K$ distribution $p \rightarrow N_{K}(p)$ is involutive (in fact autoparallel), so it is completely integrable on $G_{K}$ and the leaves of the foliation it defines on $G_{K}$ are totally geodesic (this argument adapted from [1]).

We are now ready to prove Theorem 1 .

Proof of Theorem 1. Let $\gamma:[0, c) \rightarrow L$ be a geodesic segment in $L$. It suffices to show that $\gamma$ can be extended, as a geodesic of $L$, over the half-line $[0, \infty)$. Suppose this cannot be done, and that $\gamma$ as given is maximal. Since $M$ is complete, $\gamma$ can be extended as a geodesic $\tilde{\gamma}$ of $M$. Since $L$ is totally geodesic in $M$, it follows that $\tilde{\gamma}(c)=\tilde{p}$ is not in $G_{K}$. But this means that $\mu_{K}(\tilde{\gamma}(c))>m$. We show that to be impossible.

We make the convention that $i, j, k(1 \leq i, j, k \leq m)$ shall be nullity indices, 
$a, b, c(m+1 \leq a, b, c \leq n)$ nonnullity indices, and $I, J, K(1 \leq I, J, K \leq n)$ unrestricted indices (here $n=\operatorname{dim} M)$. Now let $\eta=\left(y^{1}, \ldots, y^{n}\right)$ be a Frobenius coordinate system on a neighborhood $V$ of $\gamma(0)=p$. We can further assume that $\eta(p)=(0, \cdots, 0)$, the origin in $R^{n}$, and that $\left(\partial / \partial y^{1}\right)_{p}=\gamma^{\prime}(0)$, $\left(\partial / \partial y^{i}\right) \in N_{K}$ on $V$, and $\left(\partial / \partial y^{a}\right)_{p} \in N_{K}^{\perp}(p)$, the orthogonal complement of $N_{K}(p)$.

Nowl et $\sum$ be the slice of $V$ determined by $y^{i}=0$, and let $E=\left(E_{1}, \cdots, E_{m}\right.$, $\cdots, E_{n}$ ) be a $C^{\infty}$ orthonormal-frame field on $\sum$, adapted to the nullity distribution $\left(E_{i} \in N_{K}\right)$, such that $E_{1}(p)=\gamma^{\prime}(0)$ (we can assume $\gamma$ has unit speed). $\eta_{2}=$ $\left(y^{m+1}, \cdots, y^{n}\right)$ defines a coordinate system on $\sum$; set $\eta_{2}\left(\sum\right)=W \subset R^{n-m}$. Now define $F: R^{m} \times W \rightarrow M$ by $F\left(x^{1}, \cdots, x^{m}, \eta_{2}(s)\right)=\exp _{s}\left(x^{i} E_{i}(s)\right)$, where $s \in \sum$. Since $M$ is complete, $F$ is defined for all values in $R^{m}$.

Identify $R^{m} \times W$ with a subset $U$ of $R^{n}$, and let $\left(x^{1}, \cdots, x^{n}\right)$ be the natural Euclidean coordinate functions on $U$. Also set $x_{I}=\partial / \partial x^{I}$, and $U_{I}=F_{*}\left(X_{I}\right)$.

Now by definition, $F(t, 0 \cdots, 0)=\tilde{\gamma}(t)$, and $U_{1}(t, 0, \cdots, 0)=\gamma^{\prime}(t) \in N_{K}(\gamma(t))$ for $0 \leq t<c$. Let $G^{*}$ denote an open neighborhood of $\gamma[0, c)$ in the open set $G_{K}$, and $U^{*}=F^{-1}\left(G^{*}\right)$; for fixed $q \in W$ and all $\left(x^{1}, \cdots, x^{m}\right)$ such that $\left(x^{1}, \cdots\right.$, $\left.x^{m}, q\right) \in U^{*}, F$ parametrizes the nullity leaf through $F(0, \cdots, q)$ on a (leaf) neighborhood of that point, so the $U_{i}=F_{*}\left(\partial / \partial x^{i}\right)$ are nullity vector fields over $\left.F\right|_{U^{*}}$. It follows that $K_{U_{1} U_{a}}$ are identically vanishing tensor fields over $\left.F\right|_{U^{*}}$.

By Lemma 1 we have $\mathfrak{S} \nabla_{X_{1}}\left(K_{U_{a} U_{b}}\right)=0$. But the only surviving term on $U^{*}$ is $\nabla_{X_{1}}\left(K_{U_{a} U_{b}}\right)=0$, since covariant derivatives of vanishing tensor fields must vanish.

Now let $\tilde{Y}$ be a nullity vector at $\tilde{\gamma}(c)=\tilde{p}$. Parallelly translate $\tilde{Y}$ backwards along $\gamma$ (as parametrized by $\gamma(t)=F(t, 0, \cdots, 0)$ ) to get a parallel vector field $Y(t, 0, \cdots, 0)$ along $\left.F\right|_{(t, 0, \cdots, 0)}$. Then on $(t, 0, \cdots, 0), 0 \leq t \leq c$, we have $\nabla_{X_{1}}\left(K_{U_{a} U_{b}}\right) Y=\nabla_{X_{1}}\left(K_{U_{a} U_{b}} Y\right)=0$. But then $K_{U_{a} U_{b}} Y$ is parallel along $\gamma$, hence must vanish, since it vanishes for $t=c$. It follows that $Y(t)$ is a nullity vector for all $t, 0 \leq t \leq c$, so that $\mu_{K}(p) \geq \mu_{K}(\tilde{p})=m$. Hence $\mu_{K}(p)=m$ also, or $p \in G_{K}$, contrary to assumption, thus completing the proof.

\section{Examples and applications}

In this section we give some important examples of curvature-like tensors, as well as some related results on immersions.

In the first place, as proved in [7], the curvature tensor $R$ itself is an example of a curvature-like tensor. The nullity spaces of the curvature operator were defined in Chern and Kuiper [2]. More generally, any $(1,3)$ parallel tensor field $S$ satisfying i), ii), and iv) of $\S 2$ and hence $R-S$ are also curvature-like. In particular, $S_{X Y}=k(X \wedge Y)$, where $X \wedge Y$ is defined by $(X \wedge Y) Z=$ $\langle X, Z\rangle Y-\langle Y, Z\rangle X$, leads to Corollary 1 of the Introduction. If $M$ is a Kähler manifold and $S_{X Y}=(k / 4)(X \wedge Y+J X \wedge J Y+2\langle X, J Y\rangle J)$ where $J$ is the almost complex structure on $M$, then $S$ satisfies i)-iv) and is clearly parallel, being made up of parallel operators $\langle$, $\rangle$ and $J$. Hence $R-S$ is 
curvature-like, and we obtain Corollary 2 of the Introduction. The last two examples were introduced in Otsuki [8] and Gray [4].

We now give the proof of Theorem 2 of the Introduction.

Proof. We first note that by Lemma 1 of $\S 2$, the Bianchi-like identity v) can be written as follows:

$$
\Im V_{X}\left(K_{Y Z} W\right)=\Im\left(\nabla_{X}\left(K_{Y Z}\right) W+K_{Y Z} \nabla_{X} W\right)=\subseteq K_{Y Z} \nabla_{X} W
$$

for coordinate vector fields $X, Y, Z$, and $W$ on $M$. We will show that in fact $\subseteq \nabla_{X}\left(\bar{R}_{Y Z} W\right)=\varsigma \bar{R}_{Y Z} \nabla_{X} W$ on $M$ for coordinate vector fields $X, Y, Z$. Of course we identify $X$ and $I_{*} X$ as in [7]. Also note that $\bar{R}_{X Y} W=P \bar{R}_{X Y} W$ on $M$ since $P^{\perp} \bar{R}_{X Y} W=0$ by assumption, so $\nabla_{X} \bar{R}$ is indeed defined.

Given a point $p \in M$, choose local $\bar{M}$-coordinate vector fields $\bar{X}, \bar{Y}, \bar{Z}$, and $\bar{W}$ on a neighborhood $\bar{U}$ of $p$ in $\bar{M}$ such that $X=\left.\bar{X}\right|_{U}, Y=\left.\bar{Y}\right|_{U}$, etc. are local $M$-coordinate vector fields on $U=M \cap \bar{U}$.

Then we have $\subseteq P \bar{V}_{\bar{X}}\left(\bar{R}_{\bar{Y} \bar{Z}} \bar{W}\right)=\subseteq P \bar{R}_{\bar{Y} \bar{Z}} \bar{\nabla}_{\bar{X}} \bar{W}$ on $U$, using the $\bar{M}$-Bianchi identity. It follows that $\subseteq \nabla_{X}\left(\bar{R}_{Y Z} W\right)=\subseteq \bar{R}_{Y Z}\left(\bar{\nabla}_{X} W\right)$. But $P \bar{R}_{Y Z}(N)=0$ for normal vector fields $N$ on $U$ since $\left\langle\bar{R}_{Y Z}(N), V\right\rangle=-\left\langle\bar{R}_{Y Z}(V), N\right\rangle=0$ for arbitrary vector field $V$ tangent to $U$, and $P \perp \bar{R}_{Y Z} V=0$ by assumption. Thus $\bar{R}_{Y Z}\left(\bar{\nabla}_{X} W\right)=\bar{R}_{Y Z}\left(P^{\perp} \bar{\nabla}_{X} W+P \bar{\nabla}_{X} W\right)=\bar{R}_{Y Z}\left(\nabla_{X} W\right)$. It follows that $\subseteq \nabla_{X}\left(\bar{R}_{Y Z} W\right)$ $=\subseteq \bar{R}_{Y Z}\left(\nabla_{X} W\right)$ as required.

We mention here that since $D_{X Y}=\left[T_{X}, T_{Y}\right]$ by the Gauss equation (see [7]), it follows that $R(p) \subseteq N_{D}(p)$ for all $p \in M$, where $R(p)$ denotes the relative nullity Space at $p$.

We may state the following explicit result as a corollary of Theorems 1 and 2 .

Corolary 3. If $I: M \rightarrow \bar{M}$ is an isometric immersion such that $P^{\perp} \bar{R}_{X Y} Z=$ 0 for vector fields $X, Y, Z$ tangent to $M$, and $M$ is assumed complete, then the leaves of the nullity of $D$ foliation induced on the open set $G_{D}$ of minimal index of nullity (assumed $>0$ ) are complete totally geodesic submanifolds with curvature tensor $\bar{R}_{X Y}$ in the induced metric.

If $M$ and $\bar{M}$ are Kählerian, and the immersion $I$ is also Kählerian, then it is immediate that the nullity of $D$ and relative nullity distributions are both invariant under $J$; indeed, a result of $K$. Nomizu as reported in [1] shows that in this case $N_{D}(p)=R(p)$ for all $p \in M$. In any case, we obtain as a corollary of Theorems 1 and 2, or from the immersion theorem of [7], the following result.

Corollary 4. If $I: M \rightarrow \bar{M}$ is a Kähler immersion of the Kähler manifold $M$ into Kähler manifold $\bar{M}$ such that $P^{\perp} \bar{R}_{X Y} Z=0$ for all $X, Y, Z$ tangent to $M$, then the leaves of the relative nullity foliation induced on the set $G$ of minimal index of relative nullity (assumed $>0$ ) are complete totally geodesic complex submanifolds of $\bar{M}$, and are also the leaves of the distribution $N_{D}$ on $G$ since $N_{D}$ is identical with $R$.

Finally we mention two applications of global results on complete manifolds 
of positive curvature. First of all, as B. O'Neill and K. Abe have already remarked, a result of $\mathrm{T}$. Frankel on totally geodesic submanifolds of a complete Riemannian manifold of positive curvature shows that in Corollaries 1 and 2 if $2 \mu_{K} \geq n, n=\operatorname{dim} M$, then $\mu_{K}=n$ and $M$ has constant (holomorphic) curvature. Another result of this type is the following. A recent result of Gromoll and Meyer [5] states that in a complete noncompact Riemannian manifold $M$ of positive sectional curvature there are no compact totally geodesic submanifolds. Now by the well-known result of S. B. Myers a complete totally geodesic submanifold of positive curvature bounded away from zero would be compact. It follows, for example, that Corollary 1 with $k>0$ is vacuous (i.e., $m=0$ ) when $M$ is noncompact with positive sectional curvature.

\section{Bibliography}

[1] K. Abe, Relative curvatures and some applications to submanifolds in space forms, Doctoral thesis, Brown University, 1970.

[2] S. S. Chern \& N. Kuiper, Some theorems on the isometric imbedding of compact Riemannian manifolds in Euclidean space, Ann. of Math. 56 (1952) 422-430.

[ 3 ] Y. H. Clifton \& R. Maltz, The K-nullity spaces of the curvature operator, Mich. Math. J. 17 (1970) 85-89.

[ 4 ] A. Gray, Spaces of constancy of curvature operators, Proc. Amer. Math. Soc. 17 (1966) 897-902.

[ 5 ] D. Gromoll \& W. Meyer, On complete open manifolds of positive curvature, Ann. of Math. 90 (1969) 75-90.

[6] R. Maltz, The nullity spaces of the curvature operator, Cahiers Topologie Geom. Différentielle 8 (1966) 1-20.

[7] - Isometric immersions into spaces of constant curvature, Illinois J. Math. 15 (1971) 490-502.

[8] T. Otsuki, Isometric imbedding of Riemann manifolds in a Riemann manifold, J. Math. Soc. Japan 6 (1954) 221-234.

UNIVERSITY OF MONTREAL 
